\title{
A Memetic Study of Internet Buzzwords
}

\author{
Haiming Zhou ${ }^{1,2}$ \& Tingting $\mathrm{Li}^{1}$ \\ ${ }^{1}$ School of Foreign Languages, Yancheng Teachers University, Yancheng, Jiangsu, China \\ ${ }^{2}$ School of Foreign Languages, Soochow University, Suzhou, Jiangsu, China \\ Correspondence: Haiming Zhou, School of Foreign Languages, Yancheng Teachers University, Yancheng, \\ Jiangsu, P. R. China. E-mail: yczhm@139.com
}

Received: September 12, 2017 Accepted: October 15, 2017 Online Published: November 2, 2017

doi:10.5539/ells.v7n4p42 URL: http://doi.org/10.5539/ells.v7n4p42

\begin{abstract}
In recent years, a kind of four-character Chinese internet buzzwords like Ren Jian Bu Chai (人艰不拆) appears as a main part of internet language and attracts the attention of the linguistic field. Based on the theory of Memetics, this paper analyzes the motivation, periodicity and mechanism behind the wide imitation and transmission of Ren Jian Bu Chai as a language meme, which highlights the following three major findings: first, the pragmatic functions motivate the wide transmission and imitation of the language meme of Ren Jian Bu Chai; second, the wide spread of Ren Jian Bu Chai as a meme undergoes four stages which are assimilation, retention, expression and transmission; third, the meme of Ren Jian Bu Chai is widely transmitted and copied through two major channels, namely genotype and phenotype.
\end{abstract}

Keywords: internet buzzword, Memetics, motivation, periodicity, mechanism

\section{Introduction}

With the rapid development of computer science and technology, internet increasingly becomes one of the major means in expressing and exchanging ideas. Hence comes the phenomenon of internet language. A lot of new forms of expressions spring up, including internet buzzwords. In recent years, one type of internet buzzwords, composed of four characters, comes to occur and catch on in Chinese. Among these buzzwords, Ren Jian Bu Chai (人艰不拆) is a typical example. Since its birth on the internet, it has brought about great popularity. After this, a great many four-character internet buzzwords appear and increasingly come into wide use in daily communication. In spite of its popularity, this linguistic phenomenon causes varied and even controversial interpretations among different social groups. Some people advocate it as a creative and efficient way of expressing oneself with fun, humor and their own attitudes, while others object to this way of communication, holding the belief that it tends to incur possible ambiguities and other potential negative influences. Against this background, this study is undertaken to investigate internet buzzwords from the perspective of Memetics (Dawkins, 1976; Gatherer, 1998; Deacon, 1999; Blackmore, 1999; He \& He, 2003; He, 2005; Chen, 2006; Xie \& $\mathrm{He}, 2007 ; \mathrm{He} \& \mathrm{Li}, 2012$ ), aiming to account for the motivation, periodicity, and mechanism behind this special kind of language use.

This study is worth undertaking for the following two reasons. First, this study can to some degree help improve the communication between different communities especially the elderly and the young. The realization of human communication finds its basis on life experiences. Consequently, the elderly and the young form distinct viewpoints toward internet communication. Since internet is one major medium of modern communication and the young are likely to have easier and better access to this form of communication, young people become the mainstream users of internet language and help accelerate the spread of internet buzzwords a lot. However, the elderly may get confused toward the actual meanings of internet buzzwords, refusing to accept this kind of newborn thing in that they have relatively less and poorer access to the internet. As a result, a communication gap between the young and the elderly slowly forms due to their different life experiences and cognitive styles in understanding this new way of expression. To reduce or even eliminate hostile attitudes of the elderly, studies should be carried out to help them better understand the internet language and gradually find a way to improve the communication. Second, it is of great significance to help people have a better and deeper understanding of the history of language development. For social and historical reasons, language never stops its process of development. And each part of this process has its own features. For example, in ancient times, people used a 
classical way of expression like that adopted by Shakespeare. However, nowadays internet buzzwords become a main part of language use especially the four-character ones. As one of the features at a certain time, the analysis of its pragmatic motivation, periodicity and mechanism will greatly help understand language development as it is of great help to the comprehension of a feature of language development.

\section{Literature Review}

\subsection{Definition of Four-character Internet Buzzwords}

In Chinese, four-character internet buzzwords are different from four-character idioms though their names look alike. Four-character idioms (chéngyǔ in Chinese, also called Chinese idioms) are a type of Chinese idiomatic expression, most of which consist of four characters. These four-character idioms are often considered as fixed in form and meaning. Because the meaning of four-character idiom is often intimately related to certain historical facts from which it was derived, people usually cannot obtain its meaning simply by putting together the meanings of each character. For example, the idiom Bei Gong She Ying (杯弓蛇影) is often used to describe unnecessary suspicions or a person who is overly suspicious. Literally it means "mistaking the reflection of a bow in the cup for a snake", while figuratively it means "to be frightened by something that does not exist".

However, four-character internet buzzwords do not necessarily relate to historical events. From the perspective of lexicology, four-character internet buzzwords can be regarded as a kind of coinage. Coinage, also called neologism, is one important way of word formation. By means of coinage, people can invent or create new words with the existing words, along with which usually comes a new invention or discovery, like Kodak, Clone, AIDs, etc. There are several ways to create new words, one of which is abbreviation. Nevertheless, the abbreviation of four-character internet buzzwords is different from other words created from normal abbreviation. The abbreviation of four-character internet buzzwords particularly means the abstraction of the first characters from four Chinese words, set phrases or even sentences.

In this study, Ren (人) Jian (艰) Bu (不) Chai (拆), as a vital symbol of four-character idioms, is abbreviated form the sentence "Life is already so hard that some lies in life are better not exposed" (人生已经如此的艰难, 有些事情就不要拆穿). The underlined four characters are abstracted to form a new word which highly condenses the meaning of the original sentence. As for the originator of the sentence, there are two versions. One is Yoga Lin, a male singer from Taiwan. The sentence is one of the lines in the song of Telling lies. The other is an anonymous netizen, who said this as a reply to one blog article written by Tsai Kangyong, a famous host in Taiwan. The netizen coined this idiom to describe the contrastive of the slender and the fat. When the fat show their melancholy, people usually regard it as a sign of hunger. It is used to describe one's lack of courage to confront a hard truth.

\subsection{Previous Studies}

Since Chinese four-character internet buzzwords just appeared for a relatively short time, there are still not that many researches concerning this phenomenon. However, many studies about internet buzzwords were conducted. Various researches have been carried out to study them. Yin Hui (2013) probes into the translation methods of internet buzzwords on the basis of Nida's functional equivalence theory. The conclusion drawn is that literal translation, free translation and transliteration can be used in a comprehensive fashion to help foreigners understand the social development and culture of the country. Zhu Yiran (2013) explores the correct attitudes toward the internet buzzwords and proposes that calm attitude should be kept in face of the appearance of a word or phenomenon to study the inner social problem and public needs. Shi Xiaoni (2014) analyzes the forms, causes and possible effects of the popularization of internet buzzwords and arrives at a conclusion that internet buzzwords and hot social events most often appear at the same time, the former reflecting the latter in the form of a curved line, and the attitudes and viewpoints of internet users are expressed in a humorous manner. $\mathrm{Hu}$ Qingqing (2014) believes that internet buzzwords reflect the evolution of social culture and concludes that they reflected the real emotion of the society. These previous researches provide excellent viewpoints to dissect internet buzzwords.

A variety of previous researches have been conducted and they contribute a lot to revealing the ways of translation, cultural influences and the social attitudes toward internet buzzwords. The perspectives mentioned above are significant to the study of Chinese four-character internet buzzwords. However, the researches usually focus on one single point or another while ignoring the genetic mechanism beneath this linguistic phenomenon.

\subsection{Perspective of this Study}

There is no denying that previous researches provide excellent viewpoints to the investigation into internet buzzwords from the aspects of translation, propagation and cultural meanings. Their perspectives are various and 
studies are wide, and usually they regard the internet buzzwords as a whole for studies. However, because of this, there are limitations among them. The most obvious limitation is that these studies ignore the peculiarity of certain kinds of internet buzzwords. Furthermore, they look into internet buzzwords from a perspective which will cause that the analysis is not deep enough to explain it. Last but not the least, they fail to treat this kind of phenomenon from the perspective of linguistics and this needs studies to be done to make up for it.

Thus in this paper, the pragmatic motivation, periodicity and mechanism of the wide transmission and imitation of the Chinese four-character internet buzzwords, Ren Jian Bu Chai, will mainly be analyzed which may help people better and deeper comprehend the phenomenon of extensive imitation and transmission of Ren Jian Bu Chai after its popularity. Besides, Memetics is used as a theory foundation to analyze the mechanism which is to be studied in this thesis trying to make some effort to make up for the limitations of researches and deepen the public understanding of it.

\section{Theoretical Foundation}

\subsection{Key Terms in Memetics}

\subsubsection{Meme and Memetics}

Memetics is a newly developed theory which aims to account for the evolutionary mechanisms of culture from the perspective of Darwinism. The crucial concept of Memetics is meme, a term coined by Dawkins (1976), the ethologist and evolutionary biologist. According to Dawkins (ibid, p. 206), gene facilitated biological evolution through the processes of self-copying and competing. It is based on this assumption that he further proposes that meme boosts cultural evolution in the similar way gene does in inheritance. Later, Dawkins (1982, p. 109) deemed that the meme was a unit of information in the brain and it played an important part as a mutating replicator in cultural evolution as analogous to a gene. Dennett (1995), from the perspective of psychology, suggested that human brain was composed of various memes. However, due to lack of enough evidence to prove the idea that the replication of the units of information in the brain governs human behavior and culture, Dawkins' theory once caused great dispute among biologists, sociologists, and scientists in other research areas. In fact, Dawkins did not intend to construct a comprehensive theory of Memetics in The Selfish Gene, but rather created the term meme in a speculative manner. Therefore, researchers began to make their own interpretations of the meme and developed Dawkins' main assumptions. In China, He Ziran (2005) is the first to introduce the theory of Memetics and combines it with pragmatics. Inspired by the work made by He and his colleagues (He \& He, 2003; He, 2005; Chen, 2006; Xie \& He, 2007; He \& Li, 2012), relevant studies have been carried out, which indicates the applicability of Memetics in discourse analysis.

Foreign studies concerning Memetics can be divided into four groups, namely the view of information processing (Dennett, 1995), the view of thought contagion (Gatherer, 1998), the view of cultural evolution (Gabora, 1995), and the semiotic point of view (Deacon, 1999). He Ziran (2005) concluded that in order to define the term meme, two periods are involved. On the one hand, meme is considered as a unit of cultural imitation like tunes, rhythms, melodies, fashions, modern slogans, etc. On the other hand, meme is seen as a replicator in people's minds, like in real life, phrases, music, gestures, etc. In the real world, the working forms of meme include vocabulary, music, image, even gestures and facial expressions.

In a word, meme can be interpreted in several ways. But its core feature remains that it has great power in affecting others in language use, namely imitation and copying. According to He Ziran (2005), meme works like a kind of virus that can infect others' mind and can be passed on among people, different speech communities and even human generations.

\subsubsection{Language Meme}

In the point of view of He Ziran (2005), language itself is a meme manifest in words, phrases or contexts. A language meme includes not only its form but also the things behind it, including thoughts, believes, or intentions and so on. It is safe to say that if a word is a meme, it is a language meme. Language memes can be classified according to different criteria.

In terms of the form language memes take, we have single memes and meme complexes. Memes that occur and work in isolation are called single memes. Meanwhile, memes can also assist each other and be combined in a close relation to form units or complexes. The reason why meme complexes are formed is that "there are fierce competitions among different memes, similar memes may work mutually to form a stronger unit to achieve better circulation" (He \& He, 2003, p. 202).

In terms of the effects language memes exert, we have beneficial memes and harmful memes. Memes can have either positive or negative influence on our life and society. But in general, there are more benefits than harms 
that memes bring about. The typical manifestation of harmful memes lies in the spreading of negative social phenomena, like violence, stealing, kidnapping, drug abusing, etc.

In terms of the duration language memes last, we have powerful memes and weak memes. Powerful mems refer to those that have stronger ability to be copied, wider range to be spread, and longer time to circulate. In contrast, those have fewer chances to be or never be replicated are named weak memes or dead memes.

\subsection{The Theory of Memetics}

From above we have known that Memetics is a theory founded on an analogy with Darwinian evolution, exploring the replication, spread and evolution of memes. In this section, the stages and ways of meme transmission and imitation will be briefly reviewed.

\subsubsection{Stages of Meme Transmission}

The ability of a meme's replication makes it be transmitted and imitated. Heylighen (1998) claimed that a meme underwent four stages, namely assimilation, retention, expression and transmission. Each successful meme must transmit through these four stages. It must arouse audience's attention, break into their memory and infect them and finally get transmitted.

Assimilation means that successful memes must be able to infect a new host. Retention means that memes must remain in the memory for some time. Expression means that in order be communicated memes must be expressed as a physical entity. Transmission means that the expression of memes is transmitted via a physical medium or carrier.

\subsubsection{Ways of Meme Transmission and Imitation}

Gene is one of the most significant research results in 20th century which presented a tool profoundly to the explanation and study of the development of species. The most important characteristic of gene consists in its function of copying which makes species reproduce. It is not the gene that explains and illustrates the development of species but its ways, method and process of coping which leave people a better and deeper comprehension of human species. So crucial the gene is, and so is meme. Meme is the unit of cultural transmission which influences language a lot. Just like gene's ways, methods and process of reproducing are of great significance; those of memes are also of vitality. In He Ziran's (2005) conclusion, there are mainly two ways, in other words, forms of transmitting and duplicating a language meme, which are memetic genotype and memetic phenotype.

For genotype, it refers to the way of transmission and imitation of a meme when the same core content is inherited in various forms. Moreover, this kind of way owns its sub-ways. Two ways can be included in the memetic genotype. Firstly, the same information is directly transmitted and used under suitable conditions. In a word, quotation is used to transmit a meme. Also, the other way here is transmitting a language meme with the same information but various forms. According to He Ziran (2005), this way means using different expressions to show the same meaning.

For phenotype, it indicates that the identical set pattern is followed by different contents. Moreover, there are three sub-types of ways being included. To begin with, transmitting a meme through the same pronunciation but different meanings is one way of three. Then it comes to the same form but different contextual meanings. In the end, there is a way of same pattern but disparate senses, which means the structure and pattern of a meme do not alter, but the content of a meme changes.

\section{Memetics in Analyzing Internet Buzzwords}

According to He Ziran (2005), when a thought or a kind of information pattern appears, it can only be called a meme when it is widely transmitted and imitated. Nowadays, internet has become a main tool to accomplish communication. Internet buzzwords are used nearly everywhere, especially the four-character ones whose typical example is Ren Jian Bu Chai. Since it came into being, it has gained great popularity and imitation. It is used not only on micro-blogs for replication to some blogs, but also admitted into the word list of internet cyclopedia. Moreover, it is used as titles in newspapers to attract more attention. And then internet users create a series of internet buzzwords alike imitating it and these words also get public attention and use. These all indicate that Ren Jian Bu Chai is a meme because it has been widely transmitted and imitated. The phenomenon that so many four-character internet buzzwords are created and used imitating Ren Jian Bu Chai is a reflection and evidence of a successful meme.

\subsection{Pragmatic Motivation}

As He Ziran (2005) claimed, some memes had great power to be transmitted and imitated, while others did not. 
The key factor to judge the power of memes is their pragmatic ability. In other words, their transmission and imitation largely depend upon their ability to adapt to different situations and their positive influences on communication. Ren Jian Bu Chai has great vigor in its adaption to different situations and to a great extent it promotes communication since it came into being. In detail, it is used in different media, such as micro-blogs, newspapers, advertisements, TV programs, etc. Moreover, it is used in various situations. To transmit information and ideas, Ren Jian Bu Chai expresses the experiences about life. Take a look at the following dialogue:
A: 那个美女在看我哦! (The beauty is looking at me!)
B: 不是, 她在看广告牌。(No, she is staring at the billboard.)
A: 人艰不拆。(Life is already so hard that some lies in life are better not exposed.)

It reflects the speaker's experience about the physical world and the inner world. To show status, Ren Jian Bu Chai indicates a young community which is not only about age, but also about attitude to the world. To express affections, Ren Jian Bu Chai conveys the ironic ideas about hard truths of the world. For example, a young man invited his girlfriend to dinner, but later unfortunately found that his money had been left at home. In order to reduce the embarrassment, he may reply with the four character idiom internet buzzword Ren Jian Bu Chai. Furthermore, it can also be used to fulfill the need of entertainment. Ren Jian Bu Chai is an internet buzzword which is endowed with novelty and interesting features. In conclusion, Ren Jian Bu Chai can be used in a variety of situations which help people communicate. And this is the pragmatic meaning of it which gives it possibility to be widely transmitted and imitated as a meme.

\subsection{Transmission Periodicity}

As is mentioned above, the transmission of memes underwent four stages, namely assimilation, retention, expression and transmission (Heylighen, 1998). In this section, the transmission periodicity of Ren Jian Bu Chai will be examined based on this model.

\subsubsection{Assimilation}

By assimilation, it is meant that before transmission, the meme must be noticed, understood, and accepted by the audience. For notice, a meme must be apparent enough to cause their attention. As a four-character internet buzzword which appeared early, Ren Jian Bu Chai, its fashion and novelty quickly catches the attention the public. People soon remembered this expression and then gradually accept it for its interesting and vivid way of expressing ironic meanings. Besides, a meme must be admitted by the audience in their own cognition system for understanding. For being understood, Ren Jian Bu Chai is abbreviated from the sentence life is so hard that people should not expose lies in life. It is composed by abstracting four key words from the sentence. Their connection can be found without any effort, so the meaning can be easily understood. Finally, the meme will be accepted by the public. In other words, the more frequently people use it, the more greatly the meme of Ren Jian $B u$ Chai will affect them.

\subsubsection{Retention}

Retention is one of the four stages after assimilation. It should be emphasized that a meme cannot work well if it fails to be kept in memory. The longer it stays in human mind, the greater the influence on their transmission and imitation will be. Ren Jian Bu Chai has been noticed, understood and accepted by people for its novelty and interesting features. Besides, as mentioned above, it has great vigor in pragmatic uses. It can be drawn that it has the potential capability of staying in human memories for a relatively long period.

\subsubsection{Expression}

Expression is of profound significance in the whole progress of meme transmission because memes need to be passed down from one individual to another. It must be an entity that one could perceive. Utterance is the most outstanding means to achieve this aim. Ren Jian Bu Chai is used by internet users, as an outcome of internet which is major medium of transmission.

\subsubsection{Transmission}

Transmissibility is fundamental for meme generation in that only through wide transmission can expressions become memes. The broader a meme is transmitted, the longer it lasts, and accordingly the stronger and more vigorous it becomes. The ways of meme transmission can be identified from different perspectives (Blackmore, 1999, p. 62). In terms of the direction, memes can be transmitted vertically or horizontally. Vertical transmission usually takes place between one generation and another; which horizontal transmission occurs within the same generation. In terms of the evolutionary mechanism, memes can be transmitted in the form of 
"copy-the-product" and "copy-the-instructions". Both ways of transmission are manifest in the wide spread of the meme of Ren Jian Bu Chai, which gets transmitted from one parasitifier to another, and the range of users will become increasingly wide.

\subsection{Mechanism behind the Transmission and Imitation}

From the view of Memetics, there are two kinds of ways to copy and spread memes. One is memetic genotype and the other is memetic phenotype.

\subsubsection{Memetic Genotype}

The genotype way of transmission and imitation refers to the way that the same information is directly transmitted and used under suitable conditions. In other words, quotation is employed in this process. For example, people use the sentence "Each coin has two sides" to express that things have both advantages and disadvantages. This is one way of the memetic genotype. For the meme of Ren Jian Bu Chai, to some degree, even when people do not apprehend it well, they use it directly and more people expose to it, remember it and then quote it again. It is a way of transmission and imitation of the language meme Ren Jian Bu Chai. When responding to a difficult question to express one's feeling of frustration, people directly recall the use of it. For example, when a person eating in the dining hall finds that his boss has more money in the card than him he may directly use Ren Jian Bu Chai to express his feeling.

For the transmission and imitation, its core form and meaning are kept in this way. After it gains more popularity, it will be more frequently transmitted and imitated in nearly the same way as a gene does in inheritance.

\subsubsection{Memetic Phenotype}

Phenotype refers to the way that memes are transmitted and imitated in the same form but with new things. There are three sub-types. The first is in the same pronunciation but with different meaning, which is rarely found in the imitation and transmission of Ren Jian Bu Chai. The second is in the same form but with different contextual meanings. It follows that the meme of Ren Jian Bu Chai can be used in different circumstances, which may express different attitudes of the speakers. Take the dialogue in section 4.1 for example, the application of the meme well reflects that the speaker tries to conceal his true feeling of awkwardness. In another situation, a young couple finally got married after getting over a lot of difficulties, about which people can also Ren Jian Bu Chai to convey their feeling of deep blessings. For Ren Jian Bu Chai, the changes of contextual meanings chiefly refer to the conversion of attitudes. Because the new meaning is added, it is no longer the same word as it first appeared as a response to a host's idea.

The last way is in the same pattern but with different senses, which is typical for the transmission and imitation of Ren Jian Bu Chai. One variety generated in this way is $X i$ Da Pu Ben (喜大普奔, abbreviated from four Chinese words, 喜闻乐见，大快人心，普天同庆，奔走相告), which is used to describe the news is so thrilling that everyone is rejoicing to spread the word. Xi Da Pu Ben was even posed in New York Times, which shows the influence of the language meme of Ren Jian Bu Chai. In other words, the language meme Ren Jian Bu Chai is imitated in the way of using the same form but with different content. Many such four-character idioms come into being thereafter, like 十动然拒, 累觉不爱, 不明觉厉, etc., which are widely employed in daily communication.

\section{Conclusion}

Based on the theory of Memetics, this paper analyzes the motivation, periodicity and mechanism behind the imitation and transmission of Ren Jian Bu Chai as a language meme, which highlights the following three major findings.

Firstly, the Chinese four-character internet buzzword Ren Jian Bu Chai can adapt to different situations and fulfill the pragmatic functions of language, which results in its wide spread and gives birth to many other similar expressions. Secondly, the periodicity of the meme of Ren Jian Bu Chai is analyzed. It goes through four stages, namely assimilation, retention, expression and transmission. Thirdly, the ways of memetic transmission and imitation are explored. Like other memes, two main types of transmission are identified in the imitation and transmission of Ren Jian Bu Chai. Exactly speaking, memetic genotype and memetic phenotype help transmit and reproduce the meme of Ren Jian Bu Chai. To sum up, the theory of Memetics presents itself as an applicable framework to account for the popularity of four-character internet buzzwords like Ren Jian Bu Chai. These findings will greatly help improve daily communication and understand the causes and mechanisms of language development. 


\section{Acknowledgements}

This research is jointly supported by Top-notch Academic Programs Project of Jiangsu Provincial Department of Education (PPZY2015A012), Key Project of Philosophy and Social Science Research in Colleges and Universities in Jiangsu Province (2014SJD691) and Key Project of Educational Science of Yancheng Teachers University (13YCTCJY006).

\section{References}

Blackmore, S. (1999). The Meme Machine. New York: Oxford University Press.

Chen, L. X. (2006). Memes in advertising slogans. Foreign Language Education, 27(4), 43-46.

Dawkins, R. (1976). The Selfish Gene. New York: Oxford University Press.

Dawkins, R. (1982). The Extended Phenotype. Oxford: Oxford University Press.

Deacon, W. (1999). Memes as signs. The Semiotic Review of Books, 10(3), 1-3.

Dennett, D. (1995). Darwin's Dangerous Idea: Evolution and Meanings of Life. London: Allen Lane Press.

Gabora, L. (1995). Meme and Variations: A computer model of cultural evolution. In L. Nadel \& D. L. Stein (Eds.), Lectures in Complex Systems (pp. 471-486). Boston: Addison Wesley.

Gatherer, D. (1998). Why the "Thought Contagion" metaphor is retarding the process of memetics. Journal of Memetics-Evolutionary Models of Informational Transmission, 2(2), 135-158.

He, Z. R. (2005), Memes in language, Linguistics Sciences, 4(6), 54-64.

He, Z. R., \& He, X. L. (2003). Memetics and social usage of language, Modern Foreign Languages, 26(2), 201-209.

He, Z. R., \& Li, D. M. (2013). The critical reassessment of memes and memetics by Kate Distin: A book report of The Selfish Meme, Foreign Studies, 1(3), 7-12.

Heylighen, F. (1998). What Makes a Meme Successful? Proceedings of the 15th International Congress on Cybernetics (Association Internat. de Cybernétique, Namur), 418-423.

Hu, Q. Q. (2014). Cultural decoding and ethical reflections on the internet buzzword Tuhao. Studies in Ethics, 6(4), 136-140.

Shi, X. N. (2014). On the transmission of internet buzzwords. West China Broadcasting TV, 6(12), 27.

Xie, C. Q., \& He, Z. R. (2007). Some notes on language memes. Modern Foreign Languages, 30(1), 30-39.

Yin, H. (2013). On English translation of Chinese network hot words. Journal of Jiamusi Education Institute, $5(9), 391-392$.

Zhu, Y. R. (2013). Attitudes on internet hot words and their dispelling. Journal of Shaanxi Institute of Education, 29(1), 71-74.

\section{Copyrights}

Copyright for this article is retained by the author, with first publication rights granted to the journal.

This is an open-access article distributed under the terms and conditions of the Creative Commons Attribution license (http://creativecommons.org/licenses/by/4.0/). 